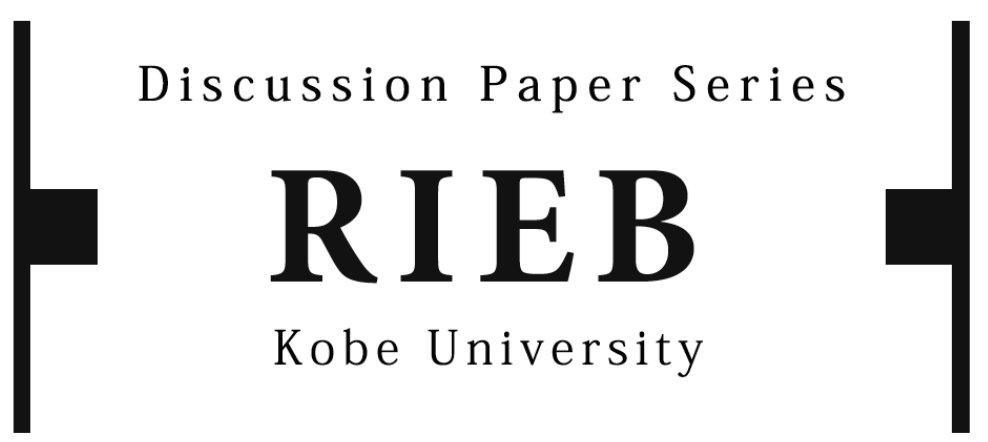

DP2014-35

Stability Analysis for Random Dynamical Systems in Economics

Takashi KAMIHIGASHI

John STACHURSKI

October 17, 2014

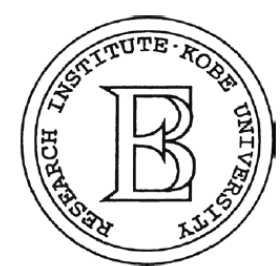

Research Institute for Economics and Business Administration Kobe University 


\title{
Stability Analysis for Random Dynamical Systems in Economics ${ }^{1}$
}

\author{
Takashi Kamihigashi ${ }^{\mathrm{a}, \mathrm{b}}$ and John Stachurskic \\ a Research Institute for Economics and Business Administration, Kobe University \\ ${ }^{\mathrm{b}}$ IPAG Business School, Paris, France \\ ${ }^{\mathrm{c}}$ Research School of Economics, Australian National University
}

October 17, 2014

\begin{abstract}
Random dynamical systems encountered in economics have certain distinctive characteristics that make them particularly well suited to analysis using the tools for studying Markov processes developed by Rabi N. Bhattacharya and his coauthors over the last few decades. In this essay we discuss the significance of these tools for both mathematicians and economists, provide some historical perspective and review some recent related contributions.
\end{abstract}

\section{INTRODUCTION}

The foremost concern within the field of economics is allocation of scarce resources among alternative and competing uses. Such resources must be allocated not only contemporaneously but also across time. Allocating resources over time necessarily involves uncertainty over possible future states of the world. These facts have led economists to maintain a deep interest in the properties of random dynamical systems.

The random processes of interest to economists have a special characteristic: Their laws and properties are generated to a large extent by the decisions of economic

\footnotetext{
${ }^{1}$ This paper was written while the second author was visiting RIEB at Kobe University as a Visiting Researcher. Our research has benefited from financial support from the Japan Society for the Promotion of Science and Australian Research Council Discovery Grant DP120100321.

Email addresses: tkamihig@rieb.kobe-u.ac.jp, john.stachurski@anu .edu .au
} 
agents - the choices of human beings. These choices are made according to a variety of concerns, such as profit maximization by firms, utility maximization by households and consumers and social welfare maximization by policy makers (should they be so inclined).

Putting humans in models is inherently problematic. Nonetheless, a broad approximation to many kinds of human behavior can be obtained by assuming that agents respond to incentives, which in the language of mathematics means that they optimize (taking into account the constraints they face, their predictions of future outcomes and perhaps their bounded knowledge and information processing capabilities). As a result, economic models almost always contain agents who optimize given their constraints, and the random dynamic systems economists analyze are determined partly by their resulting policy functions. (A "policy function" in this context usually means a map from current state to current actions). In particular, the policy functions of the agents combine with other elements of the system (equilibrium constraints, physical laws of motion, exogenous shocks, etc.) to determine the evolution of the state variables.

Policy functions are often the solution to complex optimization problems, and are typically nonlinear. (One example is threshold behavior caused by fixed costs or indivisibilities, as seen in the lumpy investment behavior of firms or oscillations in asset prices.) In many settings their exact properties are difficult to discern. If the law of motion for a given system depends on a policy function that is formally defined as the solution to a dynamic programming problem but has no analytical solution, then pinning down the exact properties of the law of motion (continuity, smoothness, etc.) becomes a difficult problem. Hence the approach to studying economic dynamics sometimes differs from methods adopted for other kinds of systems.

One particular problem associated with the issues described above is that many models either fail to be irreducible or cannot be shown to be irreducible under standard assumptions. For example, in the nonlinear models on continuous state spaces routinely treated in economics, systematic approaches to irreducibility require a considerable amount of smoothness (see, e.g., chapter 4 of [33]). It can be almost impossible to extract such fine grained information from our limited knowledge of policy functions that are defined in a formal sense but cannot be written down explicitly. 
Without irreducibility, many results from the classical theory of Markov processes (see, e.g., [33, 24]) cannot be applied. Given this scenario, it is perhaps not surprising that Rabi Bhattacharya's seminal work on Markov processes without the irreducibility assumption and his subsequent research with his coauthors on these and related topics $[3,4,14,10,7,6,9,8,12,11]$ have turned out to be ideally suited to the study of random dynamical systems in economics.

In the rest of this essay we discuss the significance of Rabi Bhattacharya's contributions through the lens of economic applications. We begin by introducing two canonical applications, extensions of which serve as workhorse models for economic research. Next we turn to theory. We also fill in some of the historical background of related work in economics, as well as subsequent developments. ${ }^{2}$

\section{BASIC ECONOMIC MODELS}

In this section we review two standard economic models that are routinely employed in economic applications (after adding in frictions or additional features that the modelers wish to study). We strip the models down to their most essential features for expositional convenience. While this eliminates some of the complications mentioned in the introduction, references are included for those who wish to dig deeper.

2.1. Optimal Growth. Foundational models in the field of growth theory analyze the dynamics of output, income, savings and consumption in a setting where growth is driven through the accumulation of productive capital [15, 34]. These models have been extended in many directions, in order to account for the role of research and development, the impact of precautionary savings, dynamics of labor through the business cycle and so forth. We present only a classical onesector optimal growth model, where a representative agent chooses a policy for consuming and saving in order to solve

$$
\begin{gathered}
\max _{\left\{c_{t}\right\}_{t=0}^{\infty}} \mathbb{E} \sum_{t=0}^{\infty} \delta^{t} u\left(c_{t}\right) \\
\text { s.t. } \quad c_{t}+k_{t} \leq y_{t}, \quad y_{t}=\xi_{t} f\left(k_{t-1}\right), \quad k_{0} \text { given. }
\end{gathered}
$$

\footnotetext{
${ }^{2}$ We focus on discrete time systems since they are more commonly observed in models of economic dynamics. Analysis of continuous time systems can be found, for example, in [13, 2].
} 
Here $u:(0, \infty) \rightarrow \mathbb{R}$ is a utility function, $\delta$ is a subjective discount factor taking values in $(0,1), f$ is a production function, $c_{t}$ is consumption, $k_{t}$ is capital (which equals savings in our simple model), $y_{t}$ is output and all variables are nonnegative. The stochastic sequence $\left\{\xi_{t}\right\}$ is taken to be IID with distribution $\phi$ having support on some subset of $(0, \infty)$. As is standard in the literature, we take $u$ to be bounded, increasing and strictly concave, with $u^{\prime}(0)=\infty$. The function $f$ is also assumed to be strictly increasing and strictly concave with $f^{\prime}(0)=\infty, f(0)=0$ and $f^{\prime}(\infty)=0$.

Under these conditions it is well known that a unique optimal savings policy $\sigma$ exists. Optimality means that if we let income evolve according to $y_{t+1}=$ $f\left(\sigma\left(y_{t}\right)\right) \xi_{t+1}$ and consume according to $c_{t}=y_{t}-\sigma\left(y_{t}\right)$, then the resulting consumption process maximizes (1) under the stated constraints [15, 27, 36]. In general no analytical expression exists for the optimal policy $\sigma$.

The slope conditions on $u$ and $f$ at zero are used partly to ensure stability and partly to generate interior choices. Regarding the first point, if $f^{\prime}(0)<\infty$, then it is possible that output converges to zero with probability one. We return to this point below. Regarding interiority, the slope conditions at zero are enough to imply that $0<\sigma(y)<y$ for all $y>0$, and, as a consequence, that $\sigma$ satisfies the Euler equation

$$
u^{\prime} \circ c(y)=\delta \int u^{\prime} \circ c[f(\sigma(y)) z] f^{\prime}(\sigma(y)) z \phi(d z) \quad(y>0)
$$

where $c(y):=y-\sigma(y)$. For a proof, see, for example, [42, prop. 12.1.24]. This equation is very useful for inferring properties of $\sigma$ and the optimal income dynamics.

To study the dynamics of the optimal process, we take $y_{t}$ as the state variable, and consider the process $y_{t+1}=f\left(\sigma\left(y_{t}\right)\right) \xi_{t+1}$. A natural state space is $(0, \infty)$ or some subinterval. Key questions are the existence and uniqueness of stationary distributions for the state variable, convergence of marginal distributions to the stationary distribution under some suitable topology, and ergodicity and central limit theorems for the time series. Answering these questions is of fundamental importance when comparing predictions with data.

2.2. Stability Arguments. In the simple version of the model we have presented, irreducibility can be established after assuming enough smoothness on $\phi$, the 
distribution of the shock process. This is because the shock $\xi_{t+1}$ appears outside the policy function in the law of motion $y_{t+1}=f\left(\sigma\left(y_{t}\right)\right) \xi_{t+1}$. However, this property is easily lost if we alter the timing or include additional complications such as correlated productivity shocks or elastic labor supply (see, e.g., [25, 17]). Although we omit such complications here, their existence implies that general stability results need to be built on top of more robust features of the dynamics.

Two such features are continuity and monotonicity. For example, consumers typically save more when income goes up. In the context of our optimal growth model, it is certainly true that the optimal savings function $y \mapsto \sigma(y)$ is continuous and increasing, and, since $f$ preserves these properties, the associated Markov process $y_{t+1}=f\left(\sigma\left(y_{t}\right)\right) \xi_{t+1}$ is both stochastically monotone and Feller $[43,15,34]$.

These properties were exploited in the first proof of stability for the model discussed above, due to Brock and Mirman [15]. They showed that the model has a unique and stable stationary distribution whenever the shocks have compact support $[a, b]$ with $0<a<b$. The same properties were also exploited in subsequent related work by Mirman and Zilcha [34] and Razin and Yahav [40]. A summary of the approach that combines monotonicity and continuity can be found in $[43] .^{3}$

However, continuity is not a robust feature that can be relied upon for stability proofs in more general cases. For example, if we drop the concavity assumption on $f$, the optimal policy can contain jumps [21, 25, 36]. Seminal work by Rabi Bhattacharya and his co-authors showed that for existence, uniqueness and stability in models such as this one sector stochastic optimal growth model, continuity of the optimal policy is unnecessary: it is sufficient to require the optimal policy to be monotone and to satisfy an appropriate mixing condition, as we discuss in Section 3.

2.3. Overlapping Generations. Another foundational class of models in economic theory are the models of production and growth with overlapping generations. Here we discuss a simple example loosely based on Galor and Ryder [23] and Wang [45]. The framework is as follows. Agents live for two periods,

\footnotetext{
${ }^{3}$ Subsequently the stability analysis was extended to the case of unbounded shocks by $[41,37$, 28, 47].
} 
working in the first and living off savings in the second. Savings in the first period forms capital stock, which in the following period will be combined with the labor of a new generation of young agents for production under the technology $y_{t}=F\left(k_{t}, \ell_{t}\right) \epsilon_{t}$. Here $y_{t}$ is income, $k_{t}$ is capital and $\ell_{t}$ is the number of young agents, all of whom supply inelastically one unit of labor. For convenience we assume that population is constant $\left(\ell_{t}=\ell=1\right)$, and set $f(k)=F(k, 1)$. Following Galor and Ryder [23, p. 362] we assume that $f: \mathbb{R}_{+} \rightarrow \mathbb{R}_{+}$has the usual properties $f(0)=0, f^{\prime}>0, f^{\prime \prime}<0, f^{\prime}(0)=\infty, f^{\prime}(\infty)=0$, as well as the extended Inada condition

$$
\lim _{k \downarrow 0}\left[-k f^{\prime \prime}(k)\right]>1 .
$$

The shocks $\left\{\epsilon_{t}\right\}$ are IID on $\mathbb{R}_{+}$according to density $\phi$.

As Galor and Ryder point out [23, Lemma 1, p. 365], restrictions on the utility function are necessary to obtain unique self-fulfilling expectations. Here we assume that young agents maximize utility

$$
U\left(c_{t}, c_{t+1}^{\prime}\right)=\ln c_{t}+\beta \mathbb{E}\left(\ln c_{t+1}^{\prime}\right), \quad \beta \in(0,1),
$$

subject to the budget constraint

$$
s_{t}=w_{t}-c_{t}, \quad c_{t+1}^{\prime}=s_{t} R_{t+1},
$$

where $s$ is savings from wage income, $c$ (respectively, $c^{\prime}$ ) is consumption while young (respectively, old), $w$ is the wage rate and $R$ is the gross rate of return on savings. Competitive markets imply that firms pay inputs their marginal factor product. Thus, the gross interest rate and wage rate are

$$
R_{t}\left(k_{t}, \epsilon_{t}\right)=f^{\prime}\left(k_{t}\right) \epsilon_{t}, \quad w_{t}\left(k_{t}, \epsilon_{t}\right)=\left[f\left(k_{t}\right)-k_{t} f^{\prime}\left(k_{t}\right)\right] \epsilon_{t} .
$$

At time $t$, households choose $s_{t}$ to maximize

$$
\ln \left(w_{t}\left(k_{t}, \epsilon_{t}\right)-s_{t}\right)+\beta \mathbb{E} \ln \left[s_{t} R_{t+1}\left(k_{t+1}, \epsilon_{t+1}\right)\right],
$$

using their knowledge of the distribution $\phi$ of $\epsilon_{t}$ to evaluate the expectations operator, as well as their current belief that next period capital stock will be $k_{t+1}$. In self-fulfilling expectations equilibrium their beliefs are realized, with

$$
k_{t+1}=s_{t}=\frac{\beta}{1+\beta} h\left(k_{t}\right) \epsilon_{t},
$$


where $h(k)=\left[f(k)-k f^{\prime}(k)\right]$. The role of condition (4) is to ensure that $h^{\prime}(0)>1$, implying that capital will not collapse to zero as long as the distribution of the shock is sufficiently favorable.

The first aim of dynamic analysis is to establish existence of a unique and stable stationary distribution for capital $\left\{k_{t}\right\}$ under reasonable assumptions on the shock process $\left\{\epsilon_{t}\right\}$. The most notable property of $h$ is monotonicity, as follows directly from concavity of the production function $f$. This makes the system amenable to analysis using the methods of Bhattacharya and Lee [3] described below.

2.4. Other Applications. We have mentioned only two simple applications. For more applications amenable to analysis using related stability conditions, see, for example, the infinite horizon incomplete market models typified by [26], stochastic endogenous growth models such as that found in [20], a wide variety of OLG models, such as those as found in [1], [39], [38] and [35], and industry models such as [16] and [19].

\section{STABILITY CONDITIONS}

In this section we discuss sufficient conditions for stability, starting with the monotonicity and "splitting" conditions introduced by Bhattacharya and Lee [3]. ${ }^{4}$

3.1. Splitting. The framework adopted by Bhattacharya and Lee [3] consists of a sequence of IID random maps $\left\{\gamma_{t}\right\}_{t \geq 1}$ on some probability space $(\Omega, \mathscr{F}, \mathbb{P})$, each map $\gamma_{t}$ sending a subset $S$ of $\mathbb{R}^{n}$ into itself, and an $S$-valued process $\left\{X_{t}\right\}_{t \geq 0}$ generated by

$$
X_{t}=\gamma_{t} X_{t-1}=\gamma_{t} \circ \cdots \circ \gamma_{1}(x)
$$

where $x \in S$ is the initial condition. The key assumption of their stability analysis is the existence of a $c \in S$ and $m \in \mathbb{N}$ such that

(S1) $\mathbb{P}\left\{\gamma_{m} \circ \cdots \circ \gamma_{1}(x) \geq c, \forall x \in S\right\}>0$; and

(S2) $\mathbb{P}\left\{\gamma_{m} \circ \cdots \circ \gamma_{1}(x) \leq c, \forall x \in S\right\}>0$.

\footnotetext{
${ }^{4}$ The work of Bhattacharya and Lee builds to some degree on earlier work by Dubins and Freedman [22] and Yahav [46].
} 
The order $\leq$ here is the usual pointwise order for vectors in $\mathbb{R}^{n}$.

Conditions (S1) and (S2) are often referred to collectively as a "splitting condition". They have a natural interpretation of mixing in an order-theoretic sense. Under this splitting condition and the assumption that all maps $\gamma_{t}$ are increasing, it was shown that the Markov process $\left\{X_{t}\right\}$ has a unique invariant distribution and is globally asymptotically stable; see $[3,5,18]$. Stability is with respect to a metric that is weaker than total variation convergence but equivalent to the Kolmogorov metric in one dimension (and implies weak convergence in higher dimensions under mild restrictions).

These simple and intuitive conditions can easily be applied to the optimal growth model described in Subsection 2.1 whenever the shock is bounded. No continuity is required, so variations that induce jumps in the policy function can also be treated. Monotonicity is known to hold, as already discussed.

Conditions (S1) and (S2) can also be used to prove stability for the overlapping generations model described in Subsection 2.3. In particular, provided that the shock distribution is chosen to be supported on a bounded subset of $(0, \infty)$, the state space $S$ for $k_{t}$ can be taken to be a bounded closed interval $\left[K_{a}, K_{b}\right] \subset(0, \infty)$. The splitting condition (S1) can then be checked by showing that, starting from $k_{0}=K_{a}$, sufficiently positive shocks can drive the state $k_{m}$ above some point $c \in\left[K_{a}, K_{b}\right]$ with positive probability. In view of monotonicity, the same shocks will drive the state above $c$ in $m$ periods from any initial condition. A proof along these lines gives (S1), and (S2) can be checked in a similar way.

Further results pertaining to the splitting conditions (S1) and (S2) were obtained by Rabi Bhattacharya and coauthors in a sequence of studies subsequent to the original paper by Bhattacharya and Lee [3]. These relate to processes that are monotone but not necessarily increasing, to the connections between splitting and classical minorization conditions, and to the implications of splitting for ergodicity and central limit theorems [4, 6, 7, 10, 12]. For example, theorem 3.1 of Bhattacharya, Majumdar and Hashimzade [12] tells us that when the overlapping generations model satisfies the splitting conditions as described above, the equilibrium capital stock process $\left\{k_{t}\right\}$ satisfies

$$
\sqrt{n}\left\{\frac{1}{n} \sum_{t=1}^{n} g\left(k_{t}\right)-\int g(x) \mu(d x)\right\} \stackrel{d}{\rightarrow} N\left(0, \sigma^{2}\right)
$$


for some $\sigma \geq 0$ where $\stackrel{d}{\rightarrow}$ means convergence in distribution. Here $\mu$ is the stationary distribution of the process (8) and $g:\left[K_{a}, K_{b}\right] \rightarrow \mathbb{R}$ is any function of bounded variation (and therefore representable as the difference between two monotone increasing functions).

3.2. Monotone Mixing. The existence of many economic models lacking irreducibility but possessing a certain monotone structure led to considerable interest in understanding the stability properties of non-irreducible random dynamical systems arising from economic models.

One well known example in the economic literature is due to Hopenhayn and Prescott [25]. They studied monotone processes that exist on a compact metric space with a closed partial order. The space $S$ is assumed to contain a least element $a$ and greatest element $b .{ }^{5}$ Monotonicity means that the stochastic kernel

$$
P(x, B):=\mathbb{P}\left\{X_{t+1} \in B \mid X_{t}=x\right\}
$$

has the property that $x \mapsto P(x, B)$ is increasing for every measurable increasing set $B$ in $S$. They require a "monotone mixing condition," which states that there exists a $c \in S$ and $m \in \mathbb{N}$ such that $P^{m}(a,[c, b])>0$ and $P^{m}(b,[a, c])>0$. This condition, combined with monotonicity, implies the splitting conditions (S1) and (S2) discussed above. Thus, although the proofs are rather different, the work of Hopenhayn and Prescott can be thought of as extending at least some of the results of Bhattacharya and Lee to abstract compact metric spaces.

3.3. Order Mixing. Recently there has been a surge of interest in developing results analogous to Bhattacharya and Lee [3] but with weaker mixing assumptions (paired, of course, with weaker conclusions in terms of uniformity and rates of convergence). To see why this might be useful, consider, for example, the stochastic optimal growth model $y_{t+1}=f\left(\sigma\left(y_{t}\right)\right) \xi_{t+1}$ and suppose now that the productivity shock $\xi$ is lognormal, say, or has any other unbounded distribution. In such a setting, the splitting conditions (S1) and (S2) are too strict. To see this, recall that the map $y \mapsto f(\sigma(y))$ is continuous and zero at $y=0$. Hence if we fix any $c>0$ and any $m \in \mathbb{N}$, the probability that $y_{m} \geq c$ conditional on $y_{0}$ can be made arbitrarily small by taking $y_{0} \downarrow 0$.

\footnotetext{
${ }^{5}$ That is, $a \leq x \leq b$ for all $x \in S$.
} 
Weaker mixing conditions maintaining an order theoretic flavor were introduced by Bhattacharya and Waymire in [14], who studied local splitting conditions in conjunction with a recurrence condition ensuring drift back to the set where splitting occurs. An alternative but related approach was suggested by Szeidl in [44].

An even weaker mixing condition was considered in [29], called order mixing. Loosely speaking, a Markov process on a partially ordered set $(S, \preceq)$ is defined to be order mixing if, given any two independent sequences $\left\{X_{t}\right\}_{t \geq 0}$ and $\left\{X_{t}^{\prime}\right\}_{t \geq 0}$ generated by the model, we have

$$
\mathbb{P}\left\{\exists t \geq 0 \text { s.t. } X_{t} \preceq X_{t}^{\prime}\right\}=1 .
$$

The initial conditions $X_{0}$ and $X_{0}^{\prime}$ are permitted to be distinct, but both processes are updated according to the same transition law. ${ }^{6}$ While order mixing is not strong enough to imply existence of a stationary distribution, it does imply uniqueness and convergence, where convergence means that if $\left\{X_{t}\right\}$ and $\left\{X_{t}^{\prime}\right\}$ are two copies of the process with different initial conditions then

$$
\left|\mathbb{E} h\left(X_{t}\right)-\mathbb{E} h\left(X_{t}^{\prime}\right)\right| \rightarrow 0 \quad \text { as } t \rightarrow \infty
$$

for any bounded measurable increasing function $h$.

Order mixing is implied by the splitting conditions (S1) and (S2). These conditions tell us that $X_{t} \preceq X_{t}^{\prime}$ occurs once ever $m$ periods with positive probability. Hence $X_{t} \preceq X_{t}^{\prime}$ eventually with probability one by the Borel-Cantelli lemma. To see that order mixing is strictly weaker than (S1) and (S2), consider two processes generated by the same Markov model, in this case $\mathrm{AR}(1)$ processes on $\mathbb{R}$ defined by $X_{t+1}=\rho X_{t}+\xi_{t+1}$ with $X_{0}=x$ and $X_{t+1}^{\prime}=\rho X_{t}^{\prime}+\xi_{t+1}^{\prime}$ with $X_{0}^{\prime}=x^{\prime}$. Here $\left\{\xi_{t}\right\}$ and $\left\{\xi_{t}^{\prime}\right\}$ are IID, standard normal, and independent of each other. While (S1) and (S2) fail, it is easy to see that $\mathbb{P}\left\{X_{1} \leq X_{1}^{\prime}\right\}$ is strictly positive, regardless of $\left(x, x^{\prime}\right)$. Hence the process is order mixing.

3.4. Order Reversing. A still weaker mixing condition was introduced in [31]. This condition combined with a techinical condition implies order mixing.

To be more precise, a Markov process $\left\{X_{t}\right\}$ on a partially ordered set $(S, \preceq)$ is called order reversing if, for any given $x$ and $x^{\prime}$ in $S$ with $x \geq x^{\prime}$, and any independent copies $\left\{X_{t}\right\}$ and $\left\{X_{t}^{\prime}\right\}$ of the process starting at $x$ and $x^{\prime}$ respectively, there

\footnotetext{
${ }^{6}$ Note that $\mathbb{P}\left\{\exists t \geq 0\right.$ s.t. $\left.X_{t}^{\prime} \preceq X_{t}\right\}=1$ must also hold by interchanging the two processes.
} 
exists a $t \in \mathbb{N}$ with $\mathbb{P}\left\{X_{t} \leq X_{t}^{\prime}\right\}>0$. In other words, there exists a point in time at which the initial ordering is reversed with positive probability. Evidently, order reversing is considerably easier to check than order mixing in applications.

A Markov process $\left\{X_{t}\right\}$ is called bounded in probability if, for any initial condition $x \in S$ and any $\epsilon>0$, there exists a compact set $C \subset S$ such that $\mathbb{P}\left\{X_{t} \in C\right\} \geq$ $1-\epsilon$ for all $t$; see, e.g., [33] or [31] for a more precise definition. If the state space itself is compact, then any stochastic process is bounded in probability. Hence this condition allows for non-compact state spaces since a Markov process on a non-compact space can be bounded in probability.

Boundedness in probability is itself not trivial to show for models like the optimal growth model discussed above, but it can be established under reasonable assumptions by exploiting the Euler equation (3). For example, if we take $w_{1}:=\left(u^{\prime} \circ c\right)^{1 / 2}$, where $c$ is the consumption policy as in (3), then some manipulations of the Euler equation lead us to

$$
\int w_{1}[f(\sigma(y)) z] \phi(d z) \leq\left[\int \frac{1}{\delta f^{\prime}(\sigma(y)) z} \phi(d z)\right]^{1 / 2} w_{1}(y) .
$$

This is a kind of drift condition, which can be used to check boundedness in probability. In this case it tells us that when income is small, the value of $w_{1}$ tends to decline (recall our assumption that $f^{\prime}(0)=\infty$ ). Since $w_{1}$ is large near zero, this means that the state moves away from zero-which is one half of boundedness in probability in this context. See $[27,36]$ for further discussion of these issues.

It has been shown [31, lemma 6.5] that for monotone processes that are bounded in probability, order reversing implies order mixing. One advantage of this approach is that, at least for monotone processes, once we have boundedness in probability and order reversing, existence of a stationary distribution requires only mild additions to the assumptions. For example, if, in addition, the stochastic kernel of the process has either a deficient or an excessive distribution (where the marginal distribution of the state is shifted up or down in the stochastic dominance ordering over one unit of time), then a stationary distribution exists, is unique and is globally stable in a topology stronger than the weak topology [31, theorem 3.1]. The essence of this fixed point argument was explored in [30] in an abstract setting. 
One can introduce still simpler mixing conditions that imply order reversing. For example, in [31] a Markov process $\left\{X_{t}\right\}$ is called upward reaching if, given any initial condition $x$ and any other point $c$ in $S$, there exists a $t \in \mathbb{N}$ such that $\mathbb{P}\left\{X_{t} \geq\right.$ c) $>0$. The process is called downward reaching if given any initial condition $x$ and any other point $c$ in $S$, there exists a $t \in \mathbb{N}$ such that $\mathbb{P}\left\{X_{t} \leq c\right\}>0$. It can be shown [31, proposition 3.2] that if a monotone process is bounded in probability and either upward or downward reaching, then it is order reversing. Related ideas are presented in [32].

\section{CONCLUSION}

The tools for studying possibly non-irreducible Markov processes introduced and refined over the past few decades by Rabi Bhattacharya and his coauthors have significantly raised the ability of economists to elicit sharp predictions from their models and compare them with data. Much interesting work remains to be done. For example, it seems likely that a more unified approach to the various order-theoretic mixing conditions discussed above can be obtained. Further, the relationship between the weaker mixing conditions and properties like laws of large numbers and central limit theorems are only starting to be investigated. On the applied side, economists are continuously generating interesting random dynamical systems and seeking the input of experts to determine their asymptotic properties.

\section{REFERENCES}

[1] Philippe Aghion and Patrick Bolton. A theory of trickle-down growth and development. The Review of Economic Studies, 64(2):151-172, 1997.

[2] Gopal K Basak and Rabi N Bhattacharya. Stability in distribution for a class of singular diffusions. The Annals of Probability, pages 312-321, 1992.

[3] Rabi N Bhattacharya and Oesook Lee. Asymptotics of a class of Markov processes which are not in general irreducible. The Annals of Probability, pages 1333-1347, 1988.

[4] Rabi N Bhattacharya and Oesook Lee. Ergodicity and central limit theorems for a class of Markov processes. Journal of Multivariate Analysis, 27(1):80-90, 1988. 
[5] Rabi N Bhattacharya and Oesook Lee. Correction: Asymptotics of a class of Markov processes which are not in general irreducible. The Annals of Probability, 25(3):1541-1543, 1997.

[6] Rabi N Bhattacharya and Mukul Majumdar. On a theorem of Dubins and Freedman. Journal of Theoretical Probability, 12(4):1067-1087, 1999.

[7] Rabi N Bhattacharya and Mukul Majumdar. On a class of stable random dynamical systems: theory and applications. Journal of Economic Theory, 96(1):208-229, 2001.

[8] Rabi N Bhattacharya and Mukul Majumdar. Dynamical systems subject to random shocks: An introduction. Economic Theory, 23(1):1, 2003.

[9] Rabi N Bhattacharya and Mukul Majumdar. Random dynamical systems: a review. Economic Theory, 23(1):13-38, 2003.

[10] Rabi N Bhattacharya and Mukul Majumdar. Random Dynamical Systems. Cambridge University Press, 2007.

[11] Rabi N Bhattacharya and Mukul Majumdar. Random iterates of monotone maps. Review of Economic Design, 14(1-2):185-192, 2010.

[12] Rabi N Bhattacharya, Mukul Majumdar, and Nigar Hashimzade. Limit theorems for monotone Markov processes. Sankhya A, 72(1):170-190, 2010.

[13] Rabi N Bhattacharya and S Ramasubramanian. Recurrence and ergodicity of diffusions. Journal of Multivariate Analysis, 12(1):95-122, 1982.

[14] Rabi N Bhattacharya and Edward C Waymire. An approach to the existence of unique invariant probabilities for Markov processes. Limit theorems in probability and statistics, 2002.

[15] William A Brock and Leonard J Mirman. Optimal economic growth and uncertainty: the discounted case. Journal of Economic Theory, 4(3):479-513, 1972.

[16] Antonio Cabrales and Hugo A Hopenhayn. Labor-market flexibility and aggregate employment volatility. In Carnegie-Rochester Conference Series on Public Policy, volume 46, pages 189-228. Elsevier, 1997.

[17] Yiyong Cai, Takashi Kamihigashi, and John Stachurski. Stochastic optimal growth with risky labor supply. Journal of Mathematical Economics, 50:167176, 2014.

[18] Santanu Chakraborty and B.V. Rao. Completeness of Bhattacharya metric on the of probabilities. Statistics $\mathcal{E}$ Probability Letters, 36:321-326, 1998. 
[19] Thomas F Cooley and Vincenzo Quadrini. Financial markets and firm dynamics. American Economic Review, pages 1286-1310, 2001.

[20] Paul A De Hek. On endogenous growth under uncertainty. International Economic Review, 40(3):727-744, 1999.

[21] W Davis Dechert and Kazuo Nishimura. A complete characterization of optimal growth paths in an aggregated model with a non-concave production function. Journal of Economic Theory, 31(2):332-354, 1983.

[22] Lester E Dubins and David A Freedman. Invariant probabilities for certain Markov processes. The Annals of Mathematical Statistics, pages 837-848, 1966.

[23] Oded Galor and Harl E Ryder. Existence, uniqueness, and stability of equilibrium in an overlapping-generations model with productive capital. Journal of Economic Theory, 49(2):360-375, 1989.

[24] Onésimo Hernández-Lerma and Jean B Lasserre. Markov Chains and Invariant Probabilities. Springer, 2003.

[25] Hugo A Hopenhayn and Edward C Prescott. Stochastic monotonicity and stationary distributions for dynamic economies. Econometrica, pages 13871406, 1992.

[26] Mark Huggett. The risk-free rate in heterogeneous-agent incompleteinsurance economies. Journal of Economic Dynamics and Control, 17(5):953969, 1993.

[27] Takashi Kamihigashi. Almost sure convergence to zero in stochastic growth models. Economic Theory, 29(1):231-237, 2006.

[28] Takashi Kamihigashi. Stochastic optimal growth with bounded or unbounded utility and with bounded or unbounded shocks. Journal of Mathematical Economics, 43(3-4):477-500, 2007.

[29] Takashi Kamihigashi and John Stachurski. An order-theoretic mixing condition for monotone Markov chains. Statistics \& Probability Letters, 82(2):262267, 2012.

[30] Takashi Kamihigashi and John Stachurski. Simple fixed point results for order-preserving self-maps and applications to nonlinear Markov operators. Fixed Point Theory and Applications, 2013:351:doi:10.1186/1687-1812-2013351, 2013.

[31] Takashi Kamihigashi and John Stachurski. Stochastic stability in monotone economies. Theoretical Economics, 9(2):383-407, 2014. 
[32] Hans G Kellerer. Random dynamical systems on ordered topological spaces. Stochastics and Dynamics, 6(03):255-300, 2006.

[33] Sean P Meyn and Richard L Tweedie. Markov chains and stochastic stability. Cambridge University Press, 2009.

[34] Leonard J Mirman and Itzhak Zilcha. On optimal growth under uncertainty. Journal of Economic Theory, 11(3):329-339, 1975.

[35] Olivier F Morand and Kevin L Reffett. Stationary Markovian equilibrium in overlapping generation models with stochastic nonclassical production and Markov shocks. Journal of Mathematical Economics, 43(3):501-522, 2007.

[36] Kazuo Nishimura, Ryszard Rudnicki, and John Stachurski. Stochastic optimal growth with nonconvexities. Journal of Mathematical Economics, 42(1):7496, 2006.

[37] Kazuo Nishimura and John Stachurski. Stability of stochastic optimal growth models: a new approach. Journal of Economic Theory, 122(1):100-118, 2005.

[38] Ann L Owen and David N Weil. Intergenerational earnings mobility, inequality and growth. Journal of Monetary Economics, 41(1):71-104, 1998.

[39] Thomas Piketty. The dynamics of the wealth distribution and the interest rate with credit rationing. The Review of Economic Studies, 64(2):173-189, 1997.

[40] Assaf Razin and Joseph A Yahav. On stochastic models of economic growth. International Economic Review, pages 599-604, 1979.

[41] John Stachurski. Stochastic optimal growth with unbounded shock. Journal of Economic Theory, 106:40-65, 2002.

[42] John Stachurski. Economic dynamics: theory and computation. MIT Press, 2009.

[43] Nancy Stokey and Robert E Lucas. Recursive Methods in Economic Dynamics (with EC Prescott). Harvard University Press, 1989.

[44] Adam Szeidl. Invariant distribution in buffer-stock saving and stochastic growth models. 2013.

[45] Yong Wang. Stationary equilibria in an overlapping generations economy with stochastic production. Journal of Economic Theory, 61(2):423-435, 1993.

[46] Joseph A Yahav. On a fixed point theorem and its stochastic equivalent. Journal of Applied Probability, pages 605-611, 1975.

[47] Yuzhe Zhang. Stochastic optimal growth with a non-compact state space. Journal of Mathematical Economics, 43(2):115-129, 2007. 